\title{
High-dose intravenous gammaglobulin therapy for acquired von Willebrand disease
}

\author{
Perry J.J. Van Genderen, Dimitri N.M. Papatsonis ${ }^{1}$, Jan J. Michiels, Jenne J. \\ Wielenga, Jeanne Stibbe and Frans J.M. Huikeshoven ${ }^{1}$
}

Departments of Haematology, and 'Gynaecology and Obstetrics, University Hospital Dijkzigt, Rotterdam, The Netherlands

\begin{abstract}
Summary: Patients with acquired von Willebrand disease may present with severe bleeding, which is usually difficult to manage. Adequate haemostasis in acquired von Willebrand disease may be achieved with the infusion of factor VIII/von Willebrand factor concentrates or with the administration of desmopressin. We report a case of acquired von Willebrand disease with severe postoperative bleeding, responding poorly to classical von Willebrand factor replacement therapy but successfully treated with high-dose intravenous gammaglobulins. This new treatment mode of acquired von Willebrand disease is discussed in the light of a critical analysis of the literature.
\end{abstract}

\section{Introduction}

Von Willebrand factor (vWF) is a large, multimeric plasma glycoprotein, synthesized in vascular endothelial cells and megakaryocytes, which plays a central role in haemostasis. It functions not only as a carrier protein for coagulation factor VIII but also has a crucial role in mediating the shear-ratedependent adhesion and aggregation of platelets at sites of injury. ${ }^{1}$ A qualitative and/or quantitative deficiency of von Willebrand factor, as is the case in patients with congenital von Willebrand disease, may likewise result in a bleeding tendency. Apart from congential von Willebrand disease, acquired cases of von Willebrand disease (AvWD) have been described. ${ }^{2,3}$ They usually occur in association with monoclonal gammopathies of undetermined significance or lymphoproliferative disorders, suggesting that the acquired deficiency of von Willebrand factor has an immunological basis, ${ }^{2,3}$ although AvWD occasionally has been described in association with solid tumours ${ }^{4,5}$ or hypothyroidism. ${ }^{6}$

Two major mechanisms have been proposed to explain the acquired deficiency of von Willebrand factor in patients with lymphoproliferative disorders or monoclonal gammopathies of undetermined significance. Firstly, antibodies inactivate functional or immunological sites of vWF or form a complex, inducing a rapid clearance from the

Correspondence: P.J.J. Van Genderen, M.D., Department of Haematology, Room L 440, University Hospital Dijkzigt, Dr Molewaterplein 40, 3015 GD Rotterdam, The Netherlands.

Accepted: 7 June 1994 circulation. ${ }^{2,3}$ Secondly, the plasma deficiency of $\mathrm{vWF}$ results from selective absorption of $\mathrm{vWF}$ to malignant cells. ${ }^{2-4}$

The treatment of AvWD is initially directe toward treatment of the primary illness as AvWDo may resolve when a remission of the underlying disorder has been achieved ${ }^{4-6}$ However, treatment of the underlying disorder may not always be possible or requires surgery, which poses a challenge to an already deteriorated haemostatic system. Adequate haemostasis in AvWD may be achieved with the infusion of factor VIII/vWF concentrates or with the administration of desmopressin. ${ }^{7}$ We report a case of AvWD with severe postoperative bleedings, unsatisfactorily responding to classical vWF replacement therapy but successfully treated with high-dose intravenous gammaglobulins (IV:Ig). This new treatment mode of AvWD is discussed in view of a critical analysis of the available literature.

\section{Case report}

A 30 year old woman was referred to the department of Haematology in September 1978 because of severe postoperative bleeding complications after a left salpingo-oophorectomy. She denied a personal or family history of a bleeding tendency. A previous surgical eye correction in 1960 and a tonsillectomy in 1965 were uneventful. Extended laboratory tests revealed a von Willebrand factor deficiency, featured by a factor VIII coagulant activity (FVIII:C) of $0.12 \mathrm{U} / \mathrm{ml}$, a von Willebrand 
factor antigen (vWF:Ag) of less than $0.10 \mathrm{U} / \mathrm{ml}$, a ristocetin cofactor activity ( $\mathrm{vWF:RCF)}$ of less than $0.12 \mathrm{U} / \mathrm{ml}$ and a prolonged Ivy bleeding time in excess of 15 minutes, which was associated with an IgG $\kappa$ monoclonal gammopathy of $8 \mathrm{~g} / 1$ (3\% bone marrow plasma cells). Mixing experiments of patient's plasma with normal pooled plasma did not reveal an inhibitor directed to either factor VIII or vWF. A right salpingectomy in May 1981 because of a ruptured endometriosis cyst and a surgical reposition in September 1982 of a fractured olecranon were uneventful after preventive cryoprecipitate infusions. The period October 1982-August 1993 was uneventful.

A re-evaluation of the monoclonal gammopathy in August 1993 revealed a progression of the monoclonal paraprotein to a multiple myeloma (a monoclonal cytoplasmic IgG $\boldsymbol{\kappa}$-positive plasma cell population, compromising $34 \%$ of the bone marrow cells; osteolytic lesions of the skull; total serum IgG $14.6 \mathrm{~g} / \mathrm{l})$. In October 1993 she presented with postmenopausal blood loss. Laboratory tests revealed an Ivy bleeding time $>15$ minutes, FVIII:C $0.87 \mathrm{U} / \mathrm{ml}$, vWF:Ag $0.38 \mathrm{U} / \mathrm{ml}$, vWF: RCF $0.11 \mathrm{U} / \mathrm{ml}$, collagen binding activity (vWF: CBA) $0.05 \mathrm{U} / \mathrm{ml}$ (normal range for all $\mathrm{vWF}$-related parameters $0.60-1.40 \mathrm{U} / \mathrm{ml}$ ) and an abnormal multimeric pattern of vWF. A diagnostic curettage under coverage of a factor VIII/vWF concentrate (Haemate $\mathrm{P}^{\mathrm{TM}}$, Behring, Germany) revealed atypical endometrial hyperplasia with focal suspicion on adenocarcinoma. The preventative administration of desmopressin was contraindicated in this patient because of hypertension. A subsequent total abdominal hysterectomy and right oophorectomy (pathologic examination of the uterus revealed no malignancy) was complicated by postoperative bleeding despite preventive infusions of Haemate $\mathbf{P}^{\mathrm{TM}}$. Adequate haemostasis could be obtained with Haemate $\mathrm{P}^{\mathrm{TM}}$ but required frequent infusions and high dosages (up to 4,000 IU every 2 hours) due to a functional inactivation and rapid clearance of infused vWF. We therefore instituted an alternative but empirical treatment with high-dose ( $1 \mathrm{~g} / \mathrm{kg}$ bodyweight) intravenous gammaglobulin (Central Laboratory for the Blood Transfusion Services, Amsterdam), which is devoid of any vWF activity, for 3 consecutive days.

\section{Results}

Infusion of 4,000 units of the factor VIII/vWF concentrate Haemate $P^{T M}$ resulted in a short-lived rise of the vWF-related activities FVIII:C, vWF:Ag, vWF:RCF and vWF:CBA, with decreased half-life times of 1-2 hours (Figure 1). Ivy bleeding times (BT) normalized transiently. In

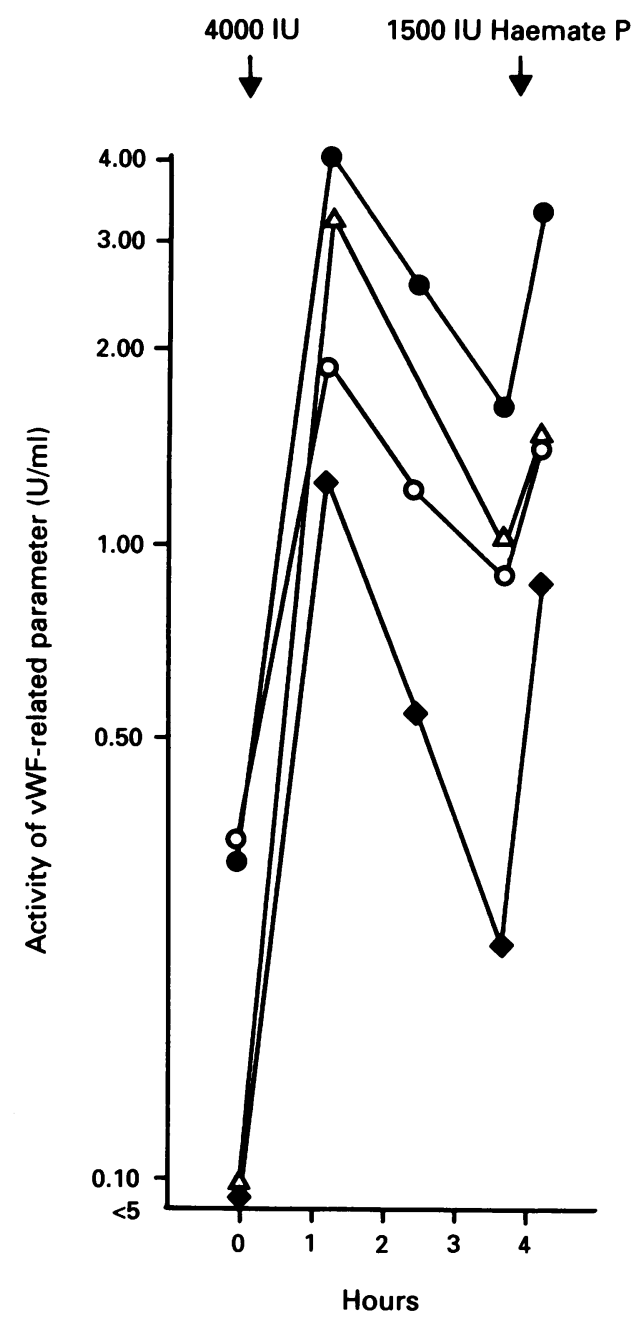

Figure 1 The effect of repeated infusions of a factor VIII/vWF concentrate (Haemate $P^{\mathrm{TM}}$ ) on von Willebrand factor-related parameters in acquired von Willebrand disease. The estimated half-life time of infused vWF is 1-2 hours (normal $8-12$ hours $^{7}$ ). $\mathrm{O}=$ FVIII:C; = vWF:Ag; $\Delta=v W F: R C F ; \diamond=v W F: C B A$. Note low functional vWF:CBA as compared with vWF:Ag concentration.

contrast, infusion of high-dose gammaglobulins induced a long-term rise of vWF-related activities and normalization of Ivy BT (Figure 2). The FVIII:C, vWF:Ag, vWF:RCF, and vWF:CBA peaked on the sixth day after institution of gammaglobulin therapy at $>4.00 \mathrm{U} / \mathrm{ml},>4.00 \mathrm{U} / \mathrm{ml}$, $4.62 \mathrm{U} / \mathrm{ml}$ and $>4.00 \mathrm{U} / \mathrm{ml}$, respectively. From day 6 all vWF-related activities slowly declined in two weeks to the clearly deficient values observed on admission. During this interval bleeding did not recur. The high preinfusion values for all vWFrelated parameters are caused by frequent dosages 


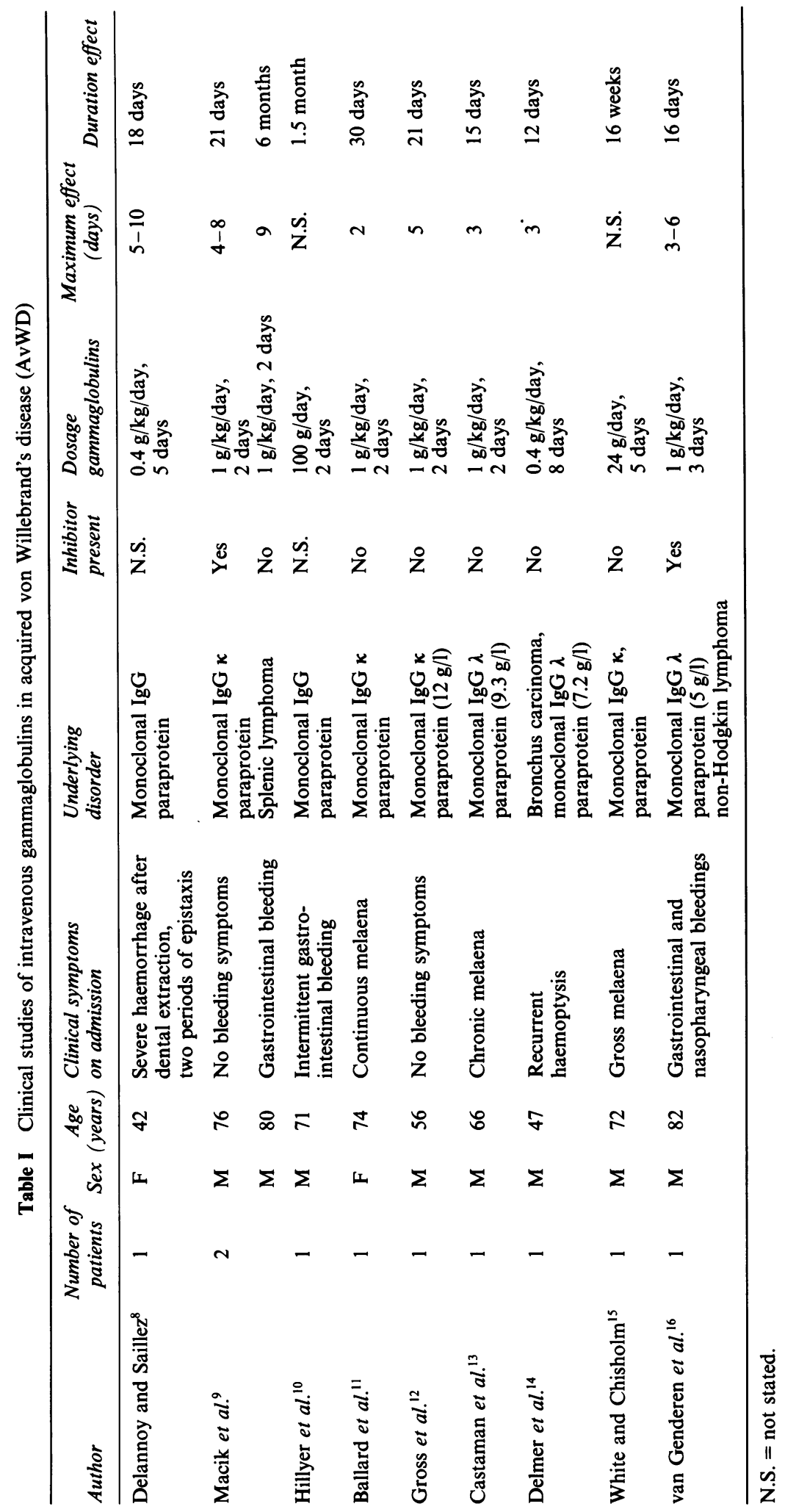



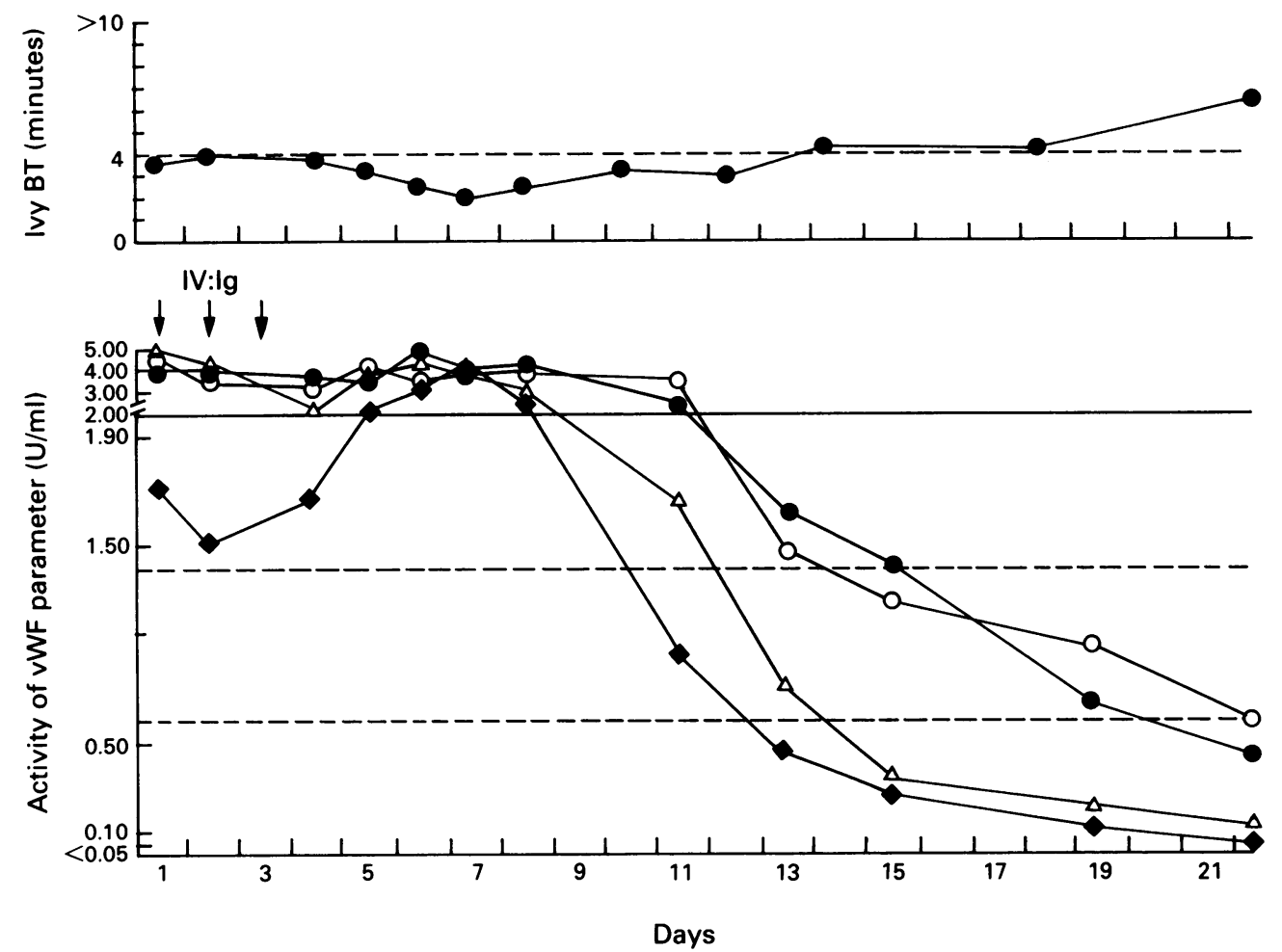

Figure 2 The effect of high-dose intravenous gammaglobulin (IV:Ig) therapy on Ivy bleeding times (BT) and von Willebrand factor-related parameters in acquired von Willebrand disease. Gammaglobulin was administered in a dose of $1 \mathrm{~g} / \mathrm{kg}$ bodyweight for 3 consecutive days (indicated by the arrows). The high preinfusion activities of the vWF-related parameters are caused by frequent infusions of Haemate $\mathrm{P}^{\mathrm{TM}}$ to secure haemostasis on the day prior to the institution of gammaglobulin therapy. The normal range for Ivy bleeding time and activities of vWF are indicated by the broken lines in the upper and lower figure, respectively. For key to symbols, see legend for Figure 1.

of Haemate $\mathrm{P}^{\mathrm{TM}}$ to secure haemostasis on the day prior to the administration of high-dose intravenous gammaglobulins.

\section{Discussion}

The patient presented illustrates the beneficial effect of high-dose intravenous gammaglobulin in the management of bleeding episodes in AvWD, insufficiently responding to infusions of a factor VIII/vWF concentrate. It is unlikely that the beneficial and long-lasting effect of high-dose gammaglobulin therapy on vWF-related activities are caused by the infusion of factor VIII/vWF concentrates on the day prior to institution of gammaglobulin therapy given the decreased halflife time of 1-2 hours of infused vWF in this patient (the normal half-life time of infused vWF is 8-12 hours $\left.{ }^{7}\right)$. Although in vitro mixing experiments of patient's plasma with normal pooled plasma, in order to demonstrate an inhibitor directed to factor VIII or VWF, did not reveal significant inactivation of the vWF-related activities, the decreased half-life time of infused $\mathrm{vWF}$ in vivo is very suggestive for the presence of a circulating anti-vWF inhibitor. This inhibitor probably forms a complex with plasma von Willebrand factor with inactivating functional sites on vWF in vivo and inducing a rapid clearance of these vWF-inhibitor complexes from the circulation by the mononuclear phagocytic systems.

Using CD-ROM assisted search of the published literature of 1988-1993, 10 additional AvWD patients (two females, eight males; ranging in age from 42 to 82 years), summarized in Table I, were identified in whom high-dose intravenous gammaglobulin therapy was successful. ${ }^{8-16}$ All patients had a monoclonal paraprotein or lymphoproliferative disorder as underlying disease, which emphasizes the presumed immunological aetiology of the acquired von Willebrand factor deficiency. An in vitro demonstrable inhibitor directed to $\mathrm{VWF}$ as a possible explanation for the acquired coagula- 
tion defect was present in only a minority of the cases. Gammaglobulin therapy was administered at a dose ranging from 0.4 to $1.0 \mathrm{~g} / \mathrm{kg}$ bodyweight for $2-8$ consecutive days. Peak values for vWF parameters were observed after 2-10 days. Gammaglobulin therapy improved the coagulation defect for 12 days to several months.

The exact mechanism of action of high-dose intravenous gammaglobulin in AvWD is not clearly understood. Several authors suggest that IV:Ig induces a blockade of the $\mathrm{Fc}$ receptors of the mononuclear phagocytic systems, which would prevent the clearance of vWF-inhibitor complexes from the circulation ${ }^{9,11-13}$ or that IV:Ig, because of its polyspecificity, may contain anti-idiotypic antibodies., ${ }^{9-14,17}$ Others propose the direct elimination of circulating immunecomplexes of vWF/anti-vWF by monomeric immunoglobulins. ${ }^{9}{ }^{13}$ Alternatively, IV:Ig may have a direct effect on the antibody-producing cells. ${ }^{9,12}$ Finally, it has been postulated that IV:Ig may directly promote the synthesis of $\mathrm{vWF}$ in vascular endothelial cells or the release of vWF from these cells. ${ }^{9}$

\section{References}

1. Ruggeri, Z. \& Ware, J. The structure and function of von Willebrand factor. Thromb Haemostas 1992, 67: 594-599.

2. Mannucci, P.M., Lombardi, R., Bader, R. et al. Studies of the pathophysiology of acquired von Willebrand's disease in seven patients with lymphoproliferative disorders or benign monoclonal gammopathies. Blood 1984, 64: 614-621.

3. Van Genderen, P.J.J. \& Michiels, J.J. Clinical manifestations and pathophysiology of acquired von Willebrand's disease in association with benign monoclonal gammapathy, multiple myeloma and lymphoproliferative disorders. In: Radl, J. \& van Camp, B. (eds) Monoclonal Gammapathies III-Clinical Significance and Basis Mechanism. EURAGE, Leiden, 1991, pp. $221-225$.

4. Facon, T., Caron, C., Courtin, P. et al. Acquired type II von Willebrand's disease with adrenal cortical carcinoma. $B r J$ Haematol 1992, 80: 488-494.

5. Richard, C., Sedano, M.C., Cuadrado, M.A., Recio, M., Hermosa, V. \& Zubizarreta, A. Acquired von Willebrand's syndrome associated with hydatid disease of the spleen: disappearance after splenectomy. Thromb Haemostas 1984, 52: $90-93$

6. Dalton, R.G., Savidge, G.F., Matthews, K.B. et al. Hypothyroidism as a cause of acquired von Willebrand's disease. Lancet 1987, i: 1007-1009.

7. Scott, J.P. \& Montgomery, R.R. Therapy of von Willebrand disease. Semin Thromb Hemastas 1993, 19: 37-47.

8. Delannoy, A. \& Saillez, A.C. High-dose intravenous gammaglobulin for acquired von Willebrand's disease. $\mathrm{Br} J$ Haematol 1988, 70: 387-389.

9. Macik, B.G., Gabriel, D.A., White, G.C., High, K. \& Roberts, H. The use of high-dose intravenous gammaglobulin in acquired von Willebrand's syndrome. Arch Pathol Lab Med 1988, 112: 143-146.

10. Hillyer, C.D., Sajer, S.A., Furie, B. \& Berkman, E.M. Severe acquired von Willebrand's disease responds to immunoglobulin infusion (abstract). Blood 1989, 74 (Suppl 1): 391.
Unfortunately, high-dose intravenous gammaglobulin therapy is not always effective in AvWD. It has occasionally been reported that patients with AvWD develop resistance toward IV:Ig therapy after repeated courses of high-dose intravenous gammaglobulin therapy. ${ }^{8}$ Furthermcre, we have recently observed a primary resistance toward IV:Ig therapy in a 63 year old male patient with a chronic lymphocytic leukaemia and associated AvWD (unpublished observation). In these cases of AvWD, both refractory to classical vWF replacement therapy and high-dose intravenous gammaglobulin therapy intensive plasma exchange ${ }^{18}$ or even extracorporeal immunoabsorption of anti-vWF antibodies ${ }^{19}$ may be considered to induce a temporary improvement of the coagulation defect at times of bleeding.

In conclusion, an empirical treatment with highdose intravenous gammaglobulin therapy should be considered in each patient with AvWD, refractory to classical cWF replacement therapy, to induce a long-term improvement of the coagulation defect.

11. Ballard, J.O., Sanders, J.C. \& Eyster, M.E. Acquired von Willebrand's disease and angiodysplasia: response to intravenous immune globulin (abstract). Blood 1989, 74 (Suppl 1) 387.

12. Gross, S., Traulle, C., Capiod, J.C. et al. Efficacy of high-dose intravenous gammaglobulin in the management of acquired von Willebrand's disease during orthopaedic surgery. $\mathrm{Br} J$ Haematol 1992, 82: 170-171.

13. Castaman, G., Tosetto, A. \& Rodeghiero, F. Effectiveness of high dose intravenous immunoglobulin in a case of acquired von Willebrand's syndrome with chronic melena not responsive to desmopressin and factor VIII concentrate. Am J Hematol 1992, 41: 132-136.

14. Delmer, A., Horellou, M.H., Brechot, J.M. et al. Acquired von Willebrand disease: correction of hemostatic defect by high-dose intravenous immunoglobulins. Am $\mathrm{J}$ Hematol 1992, 40: $151-152$.

15. White, L.A. \& Chisholm, M. Gastro-intestinal bleeding in acquired von Willebrand's disease: efficacy of high-dose immunoglobulin where substitution treatments failed. $\mathrm{Br} J$ Haematol 1993, 84: 332-334.

16. Van Genderen, P.J.J., Michiels, J.J., Bakker, J.J. \& van't Veer, M.B. Effectiveness of high-dose intravenous gammaglobulin therapy in acquired von Willebrand's disease. Vox Sanguinis 1994 (in press).

17. Sultan, Y., Kazatchkine, M.D., Maisonneuve, P. \& Nydegger, U.E. Anti-idiotypic suppression of autoantibodies to factor VIII (antihaemophilic factor) by high-dose intravenous gammaglobulin. Lancet 1984, 2: 765-768.

18. Silberstein, L.E., Abrahm, J. \& Shattil, S.J. The efficacy of intensive plasma exchange in acquired von Willebrand's disease. Transfusion 1987, 27: 234-237.

19. Uehlinger, J., Rose, E., Aledort, L.M. \& Lemer, R. Successful treatment of an acquired von Willebrand factor antibody by extracorporeal immunoadsorption. $N$ Engl J Med 1989, 320: 254-255. 